
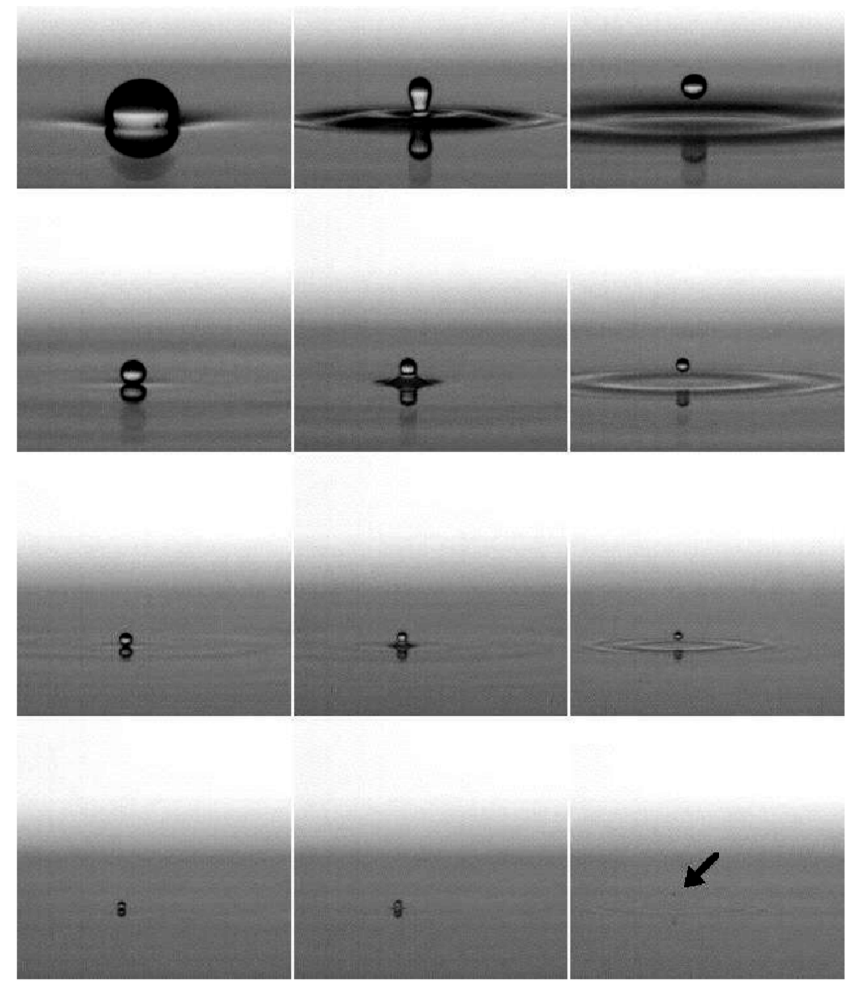

FIG. 1. A cascade of partial coalescences experienced by a soapy water droplet (enhanced online).

\section{Dancing droplets onto liquid surfaces}

N. Vandewalle, D. Terwagne, K. Mulleners,

T. Gilet, and S. Dorbolo

Université de Liège, 4000 Liège, Belgium

(Received 1 June 2006; published online 26 September 2006)

[DOI: 10.1063/1.2335905]

The first experiment settles in a container filled with soapy water. A small droplet of this liquid is gently laid down on the bath surface (Fig. 1). This droplet stays at rest for a moment on the surface, due to the drainage of a thin air film between the droplet and the bath surface. Eventually, a hole tears the film and the droplet experiences a partial coalescence. This phenomenon is mainly due to capillary waves that are generated as the air film collapses. They propagate from the bottom to the top of the droplet. The convergence of these waves at the top of the droplet is able to delay the vertical collapse in aid of the horizontal one. ${ }^{1}$ A pinch-off occurs and a daughter droplet is formed, with a radius about a half the mother droplet. This new droplet can also partially coalesce after a short rest. The main condition for partial coalescence is that capillary forces have to be the only dominant forces. Therefore, a large droplet, for which gravity forces are as much important as surface tension forces, experiences a total coalescence. The same is observed at microscales, when viscosity forces in air and water are becoming significant. ${ }^{2}$ As much as six successive partial coalescences can be observed, starting from a millimetric droplet to a droplet about 30 microns in radius.

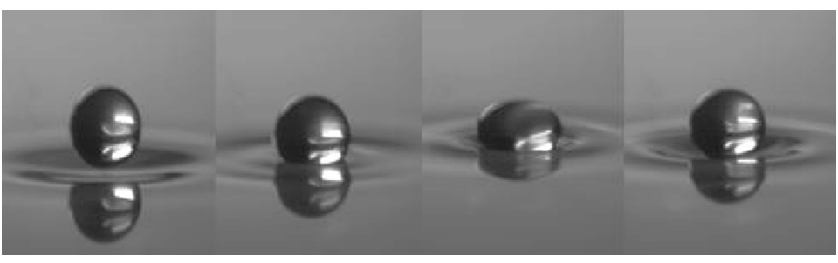

FIG. 2. A single oil droplet bouncing on a vibrated air/oil interface.

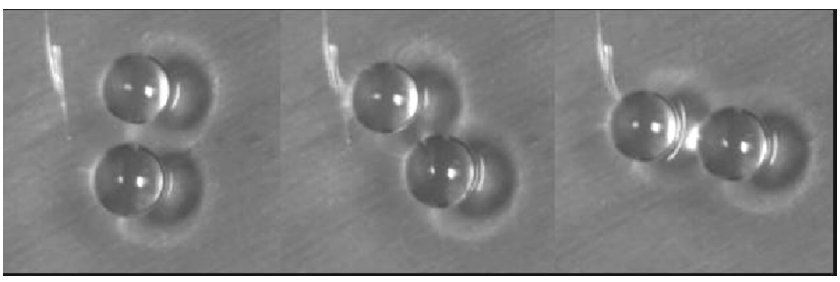

FIG. 3. Top view of two droplets orbiting around each other.

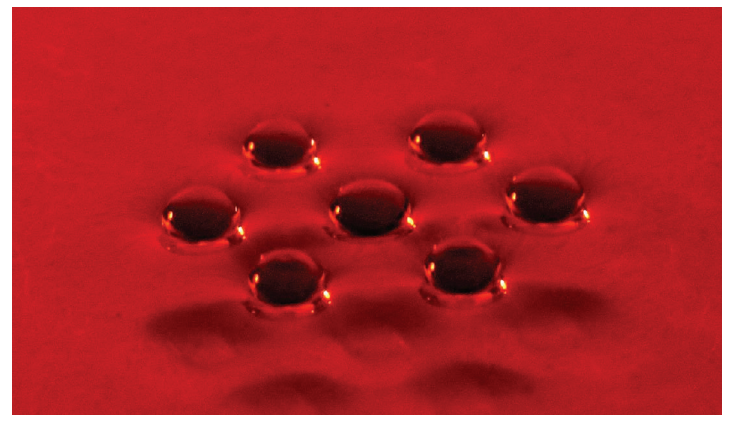

FIG. 4. Hexagonal pattern formed by seven droplets.

The second experiment consists in a similar container, filled with silicon oil (DC200-50cSt). The bath is subjected to a vertical oscillation at $75 \mathrm{~Hz}$. The vibration is set below the Faraday instability threshold. A droplet of the same fluid is laid down, like in the partial coalescence experiment. Due to the vibration, the air film below the drop is constantly regenerated: ${ }^{3}$ the drop does not coalesce for a long time (often more than a quarter of an hour); it simply bounces on the interface. The droplet experiences really high deformations during its bouncing (Fig. 2). So does the interface, where capillary waves are periodically generated. When another droplet bounces in the vicinity of the first one, the interactions between waves result in an attraction between the droplets. With appropriate initial conditions, orbiting is possible (Fig. 3). Typically, two droplets laid on the same place will stay at a finite distance (typically the capillary wavelength) from the others. Supplementary droplets take position in order to form regular patterns. For example, seven droplets adopt the traditional hexagonal lattice positions (Fig. 4).

${ }^{1}$ F. Blanchette and T. P. Bigioni, "Partial coalescence of drops at liquid interfaces," Nat. Phys. 2, 254 (2006).

${ }^{2}$ T. Gilet, K. Mulleners, J. P. Lecomte, N. Vandewalle, and S. Dorbolo, "Critical parameters for the partial coalescence of a droplet," Phys. Rev. E (submitted).

${ }^{3}$ Y. Couder, E. Fort, C. H. Gautier, and A. Boudaoud, "From bouncing to floating: Noncoalescence of drops on a fluid bath," Phys. Rev. Lett. 94, 177801 (2005). 